\title{
Performance Evaluation of Eleven-Phase Induction Machine with Different PWM Techniques
}

\author{
M.I. Masoud ${ }^{*_{a}}$ and A.S. Abdelkhalik ${ }^{b}$ \\ ${ }^{a}$ ECE Department, College of Engineering, Sultan Qaboos University, Muscat, Oman \\ ${ }^{b}$ EE Department, College of Engineering, Alexandria University, Alexandria, Egypt
}

Received 9 June 2014; accepted 4 December 2014

\begin{abstract}
Multiphase induction machines are used extensively in low and medium voltage (MV) drives. In MV drives, power switches have a limitation associated with switching frequency. This paper is a comparative study of the eleven-phase induction machine's performance when used as a prototype and fed sinusoidal pulse-width-modulation (SPWM) with a low switching frequency, selective harmonic elimination (SHE), and single pulse modulation (SPM) techniques. The comparison depends on voltage/frequency controls for the same phase of voltage applied on the machine terminals for all previous techniques. The comparative study covers torque ripple, stator and harmonic currents, and motor efficiency.
\end{abstract}

Keywords: Multiphase induction machine, Eleven-phase, Selective harmonic elimination, Sinusoidal pulse-width-modulation, WM, Single pulse modulation, Medium voltage, Square wave.

$$
\begin{aligned}
& \text { تقييم الأداء للاكينات الحث ذات الإحدى عشر طوراً لتقنيات مختلفة لتغيير عرض } \\
& \text { النبهة } \\
& \text { م. إ. مسعود و أ. س. عبد الخالق }
\end{aligned}
$$

*Corresponding author's e-mail: m.masoud@squ.edu.om 


\section{Introduction}

In high-power industrial applications such as electric ship propulsion, aircraft drives, and mining applications, high power ratings for both the motor and converter are required (Levi 2008; Levi et al. 2007; Parsa 2005; Singh 2002 and Abdelkhalik et al. 2010). However, converter ratings cannot be increased above a certain range due to semiconductor devices' power rating limitations (Levi 2008). Since adjustable speed drives require a power electronic converter for the motor supply, the number of machine phases can be unlimited (Levi 2008 and Singh 2002). Accordingly, a multiphase machine fed from a multiphase inverter drive could be a candidate for this task (Levi et al. 2007; Singh 2002). A multiphase induction machine has the same control principle as the conventional three-phase induction machine, but it is superior from a rating and fault tolerant point of view to multi-level inverters coupled with a three-phase induction machine. The multiphase machine can be fed from a square wave supply with improved performance compared to a conventional traditional induction machine fed from a six-step converter (Abdelkhalik et al. 2010; Abdelkhalik et al. 2011). The torque per ampere is improved if the number of phases is increased compared to a conventional sinusoidal pulse-width-modulation(SPWM) controlled inverter (Duran et al. 2008). The square wave supply has merit as it runs the inverter with a fundamental frequency rather than using a high switching frequency, which in turn reduces switching losses (Abdelkhalik et al. 2010). On other hand, a SPWM-controlled inverter attains higher torque per ampere for light loads. A high switching frequency is required for a SPWM to obtain the accepted waveform applied to the motor (sinusoidal nature), but using a high switching frequency is limited in medium voltage (MV) systems. Alternatively, the selective harmonic elimination (SHE) technique can be used, which satisfies low-order harmonic elimination in voltage-source inverters (Abdelkhalik et al. 2011; Epinoza et al. 2001; Zhang \& Sun 2006; Blasko 2007). While the harmonic elimination procedure might be chosen to improve the current waveform of a multi-phase inverter-fed IM drive, it increases torque pulsations (Pavithran et al. 1988).

This paper introduces a comparative study of multiphase induction machine performances when fed from different modulation techniques the SHE technique, SPWM with a low switching frequency suitable for MV applications, and single-pulse modulation (SPM) with fundamental frequency-with a focus on eleven-phase machines. Comparison was based on torque ripple, stator current, and efficiency versus load torque. Two cases were studied for this comparison. In the first case, the modulation index was kept constant $(M=1)$ while the direct current (DC) linked voltage was varied to achieve a $V / f$ constant. In the second case, the DC-linked voltage was constant while the modulation index was varied to keep the $V / f$ constant. A practical result confirmed that simulation results were introduced. The construction of the eleven-phase induction machine is given in the appendix.

The targeted outcome of this work was to identify which technique is most suitable for MV drive applications and the effect of a modulation index on the chosen technique for multiphase machines where an eleven-phase machine is employed. In light of this, the pros and cons of each technique are examined from a loading point of view. The papers is organized, after the introduction, as follows: section II describes multiphase machine modeling, section III introduces the three modulation techniques (SHE, SPWM, and SPM), section IV illustrates the performance analysis evaluation using these modulation techniques, and the paper ends with a conclusion and contributions description.

\section{Multiphase Machine Modeling}

The multi-phase induction machine can be modeled as multiple decoupled $d q$ planes by using an appropriate transformation (Toliyat et al. 1991; Pereira et al. 2006 and Libo et al. 2008). Each plane corresponds to a certain sequence. For an odd phase system with n-phases, the corresponding number of planes will be $m=1 / 2(n-$ 1 ) with one zero sequence. However, for an even number of phases, the number of decoupled 
planes is $m=1 / 2(n-2)$, with two zero sequences. The $d q$ voltage equations corresponding to each sequence can be written as:

$$
\begin{aligned}
& v_{d s k}=R_{s} i_{d s k}+p \lambda_{d s k}-k \omega_{f} \lambda_{q s k} \\
& v_{q s k}=R_{s} i_{q s k}+p \lambda_{q s k}+k \omega_{f} \lambda_{d s k} \\
& 0=R_{r k} i_{d r k}+p \lambda_{d r k}-k\left(\omega_{f}-\omega_{r}\right) \lambda_{q r k} \\
& 0=R_{r k} i_{q r k}+p \lambda_{q r k}+k\left(\omega_{f}-\omega_{r}\right) \lambda_{d r k}
\end{aligned}
$$

where

$$
\begin{aligned}
& \lambda_{d s k}=L_{s k} i_{d s k}+L_{m k} i_{d r k} \\
& \lambda_{q s k}=L_{s k} i_{q s k}+L_{m k} i_{q r k} \\
& \lambda_{d r k}=L_{r k} i_{d r k}+L_{m k} i_{d s k} \\
& \lambda_{q r k}=L_{r k} i_{q r k}+L_{m k} i_{q s k}
\end{aligned}
$$

and $k$ equals $1,3,5, \ldots 2 m-1$.

Each sequence contributes to the mechanical torque production

$$
T_{e k}=\frac{n P}{4} k\left(\lambda_{d s k} i_{q s k}-\lambda_{q s k} i_{d s k}\right)
$$

and the total torque is

$$
T_{e}=\sum_{k=1,3, . .}^{n-2} T_{e k}
$$

The transformation matrix, which transforms the phase variables into dq variables, is

$$
T=\frac{2}{n}\left(\begin{array}{cccccc}
c \theta & c \theta_{1} & c \theta_{2} & \cdots & \cdots & c \theta_{n-1} \\
-s \theta & -s \theta_{1} & -s \theta_{2} & \cdots & \cdots & -s \theta_{n-1} \\
c 3 \theta & c 3 \theta_{1} & c 3 \theta_{2} & \cdots & \cdots & c 3 \theta_{n-1} \\
\vdots & \vdots & \vdots & \vdots & \vdots & \vdots \\
c(2 m-1) \theta & c(2 m-1) \theta_{1} & c(2 m-1) \theta_{2} & \cdots & \cdots & c(2 m-1) \theta_{n-1} \\
-s(2 m-1) \theta & -s(2 m-1) \theta_{1} & -s(2 m-1) \theta_{2} & \cdots & \cdots & -s(2 m-1) \theta_{n-1} \\
1 / 2 & 1 / 2 & 1 / 2 & \cdots & \cdots & 1 / 2
\end{array}\right)
$$

This model can be used for an eleven-phase induction machine where the number of phases (represented by $n$ ) is 11 and, accordingly, the number of planes (represented by $m$ ) is 5 . This means an eleven-phase machine has eleven fundamental phases and can accept the injection of third, fifth, seventh, and ninth harmonics.

The equivalent circuit at a steady state, which is identical to a three-phase induction motor circuit per phase, was used in this study (Abdelkhalik et al. 2010). Figure 1 shows the steady-state equivalent circuit of a eleven-phase induction machine per phase for any sequence $k$.

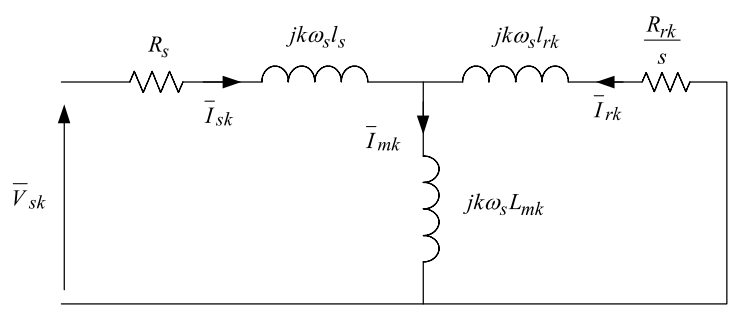

Figure 1. A steady-state equivalent circuit for any sequence $k$.

\section{Modulation Techniques: Selective Harmonic Elimination, Sinusoidal Pulse-Width-Modulation, and Sin- gle Pulse Modulation}

The eleven-phase induction machine performance varies when different modulating techniques are used. Each modulation technique provides harmonic patterns which affect the flux, torque, and efficiency of the machine. Figure 2 shows a block diagram of the drive system.

\subsection{Selective Harmonic Elimination (SHE)}

The SHE, which is an off-line modulation method, eliminates several low-order harmonics in the inverter current. The switching angles can be pre-calculated (Abdelkhalik et al. 2011). The number of eliminated harmonics determines the required number of switching angles per halfcycle. The Fourier expansion depends on the number of pulses (Duran et al. 2008), where $\mathrm{m}$ is the $\mathrm{m}^{\text {th }}$ switching angle in the $\mathrm{N}$ switching angle of $[0, \pi / 2]$ and $\mathrm{k}$ is the order of the harmonic.

SHE patterns can be achieved by solving Equation (1). For an eleven-phase machine, up to the ninth harmonic can be eliminated to have only 


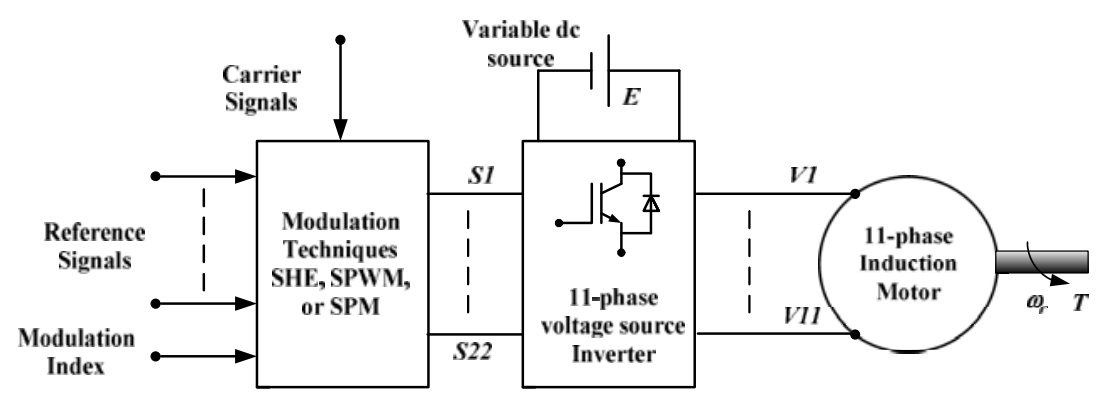

Figure 2. Block diagram of an eleven-phase drive system.

$a_{k}=\frac{4 V}{j \pi}\left[-1-2 \sum_{m=1}^{N}(-1)^{m} \cos \left(j \alpha_{m}\right)\right]$

a fundamental sequence. The switching frequency is chosen according to its suitability for MV inverters (Abdelsalam et al. 2010). The eliminated harmonics are the third, fifth, seventh, ninth, eleventh, thirteenth, and fifteenth. The thirteenth harmonic is the negative sequence for the fundamental. The elimination of the fifteenth harmonic achieves an odd number of switching angles. As there are seven switching angles, the switching frequency satisfies the MV drives. The switching angles modulation index, $M$, relation was detailed by Abdelkhalik et al. (2011). The machine was tested such that the operating frequencies were $12.5 \mathrm{Hertz}(\mathrm{Hz}), 25 \mathrm{~Hz}, 37.5 \mathrm{~Hz}$, and $50 \mathrm{~Hz}$, and the switching frequency was 375 $\mathrm{Hz}$.

\subsection{Sinusoidal Pulse-Width-Modulation (SPWM)}

The SPWM was adjusted to a $375 \mathrm{~Hz}$ switching frequency to allow a fair comparison with the SHE technique. The two-level SPWM was obtained by comparing a $375 \mathrm{~Hz}$ triangular carrier with eleven modulating sine-waves, with a $360 / 11^{\circ}$ phase shift between each. The SPWM with a $375 \mathrm{~Hz}$ switching frequency when applied to the eleven-phase machine resulted in a current free of the third, fifth, seventh, and ninth harmonics.

\subsection{Single Pulse Modulation (SPM)}

To obtain a three-level single pulse output, an
H-bridge inverter was used for each phase (Fig. 3 ). The pulse width and frequency were adjusted to meet the required $V / f$ ratio where the output was a quasi-square wave. The phase voltage obtained from the H-bridge where the positive pulse was obtained when switches 1 and 2 were on, and the negative pulse was obtained when switches 3 and 4 were on. The zero voltage intervals were obtained by either turning switches 1 and 3 on or turning switches 2 and 4 on Fig. 3 . Unlike SHE and SPWM, SPM produces stator currents containing all harmonics except for the eliminated eleventh harmonic.

\section{Performance Analysis}

To assess the performance of these PWM techniques, a concentric windings prototype eleven-phase induction motor fed from an elevenphase voltage source inverter was used to obtain both the simulation and experimental results. An Infineon TC1796 DSP (Infineon Technologies AG, Neubiberg, Germany) was used to implement practical control signals. The mechanical load was simulated using a DC generator. The appendix illustrates machine ratings and parameters referred to the stator. The simulation results were obtained using the MATLAB/Simulink platform (MathWorks, Matick, Massachusetts, USA).

Two cases were studied to compare the SHE, SPWM, and SPM techniques. In the first case, the modulationindex, was kept constant at $M=1$ while the DC-link voltage was varied to achieve a $V / f$ constant. In the second case, the DC-link voltage was constant while the modulation index varied to keep $V / f$ constant. 


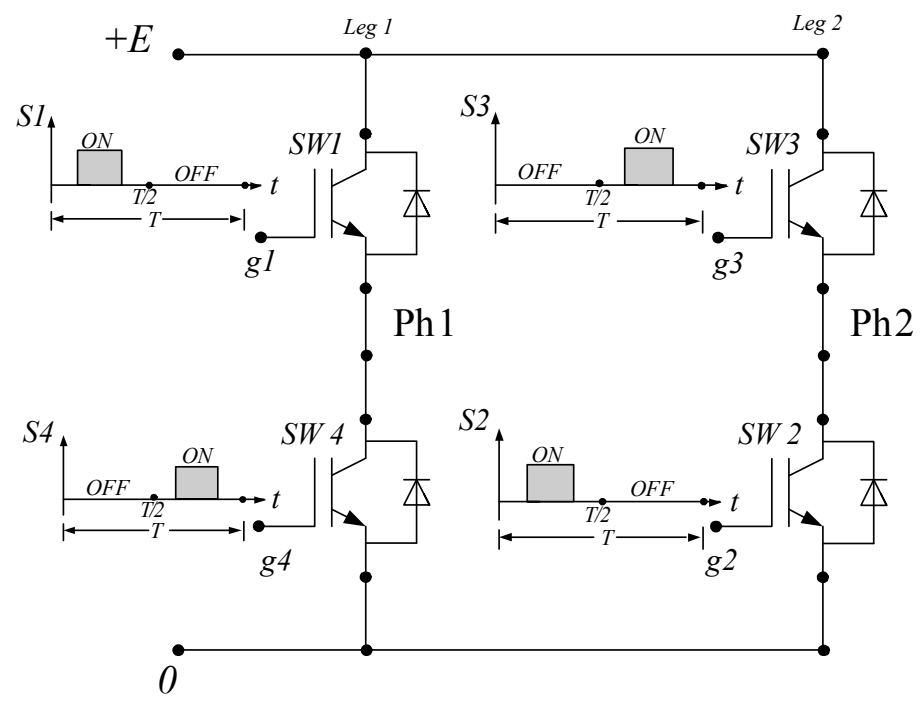

Figure 3a. Single pulse generation for an eleven-phase inverter using an H-bridge.

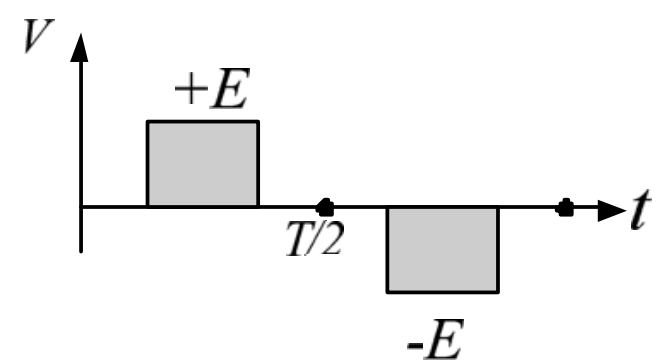

Figure 3b: The single-pulse output phase voltage of an H-bridge inverter.

For the variable DC-link, a controlled full bridge rectifier can be used but it increases costs and losses, especially in MV motors which are factors to be taken into consideration.

\subsection{Steady State Response}

Figure 4 shows the machine performance simulation results, with consideration given to load torque versus torque ripple, torque derivative peak-to-peak value, stator root mean square (RMS) current, stator fundamental current, RMS harmonic current, and motor efficiency, with a variable DC-link voltage as assessed at the rated voltage and a speed with a modulation index equal to 1 . Figure 5 shows the same performance but with a variable DC-link obtained at half the rated voltage and speed. Figure 6 gives the performance of fixed DC-link results obtained at half the rated voltage and speed.
Figure 4 shows that the SHE at $375 \mathrm{~Hz}$ has better efficiency at light loads than SPM or SPWM; however, after approximately 10 Newtons per meter $(\mathrm{Nm})$, the SPM yields a higher efficiency. Since the units of torque ripple and torque derivative peak are $\mathrm{Nm} / \mathrm{s}$, which is the same unit of power, torque ripple and torque derivative peak values implicitly represent the power lossesin the motor rotor and shaft. Reducing torque ripple and torque derivative also reduces the mechanical stresses on the rotor and shaft, thus increasing their lifespan. Figures 4 and 5 show that SPM has lower torque ripple and torque derivative peak values compared to SHE and SPWM. Thus, SPM provides a longer lifespan for a motor rotor and shaft and, consequently, it is recommended for MV motors to avoid mechanical damage that can be inflicted over a short period of time. If efficiency is the main 


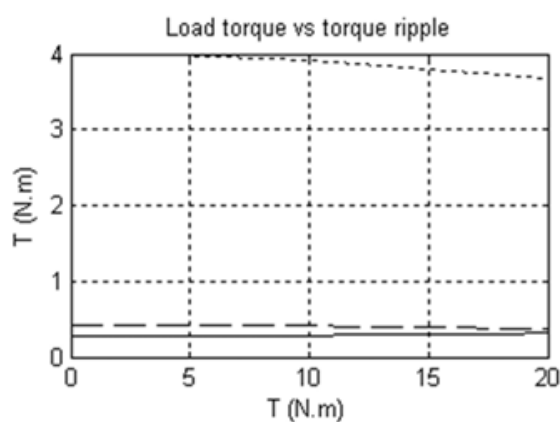

(a)

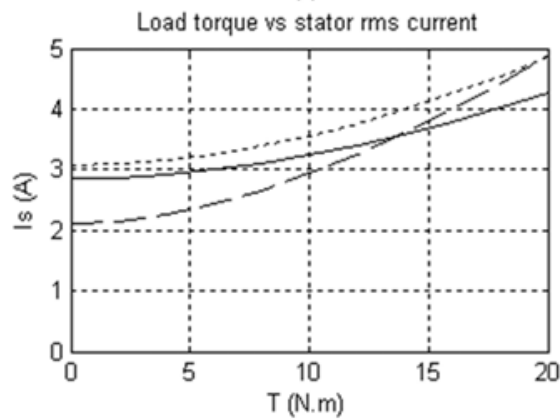

(c)

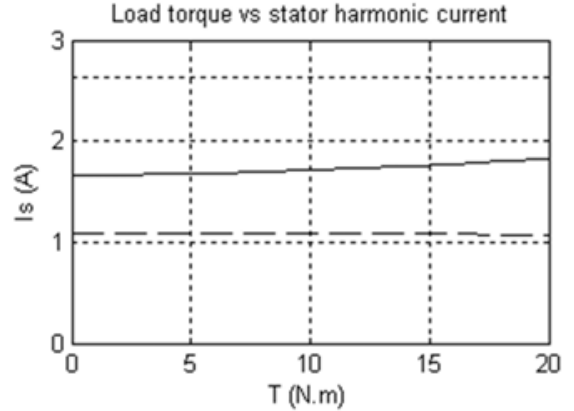

(e)

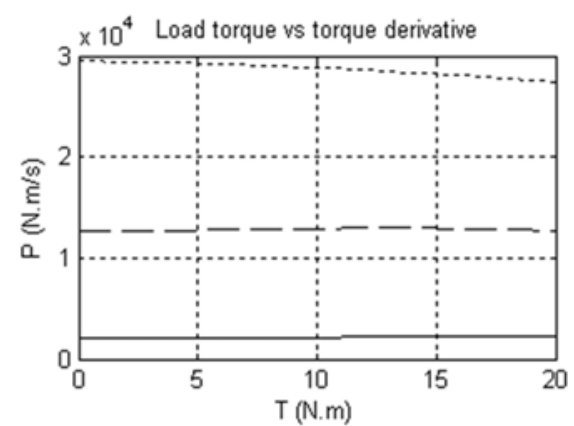

(b)

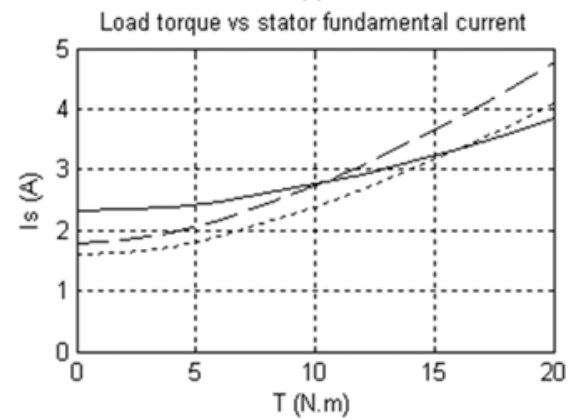

(d)

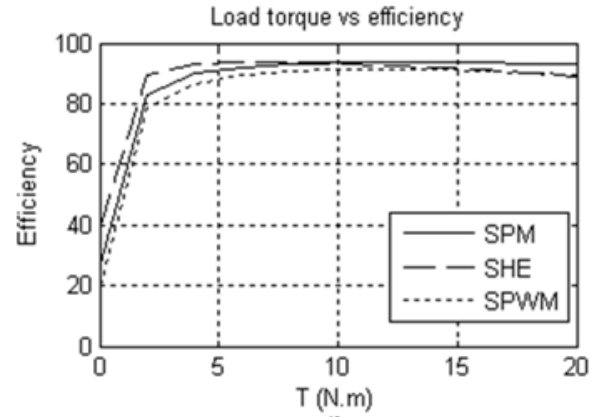

(f)

Figure 4. Variable DC-link simulation results obtained at the rated voltage and speed for load torque versus torque ripple (a), torque derivative peak value (b), stator RMS current (c), stator fundamental current (d), stator harmonic current (e), and motor efficiency (f).

concern, the motor controller can switch between SHE and SPM depending on loading conditions while taking into consideration the fact that both SHE and SPWM have higher switching losses than SPM, especially in MV drive systems. Figures 4 and 5 show the relationship between load torque and stator harmonic current. The first harmonic in both SHE and SPWM is the fifteenth harmonic which does not contribute in torque; however, the stator harmonic currents are constant. In SPM, all harmonics are included where the third, fifth, seventh, and ninth harmonics contribute to torque (Levi 2008; Abdelkhalik et al. 2010). At rated voltage, SPWM yields the highest stator RMS current but, at half the rated voltage, SPM stator RMS current is higher due to the increase in stator harmonic current.

For a fixed DC link (Fig. 6) the DC converter cost is lower which is recommended for MV 


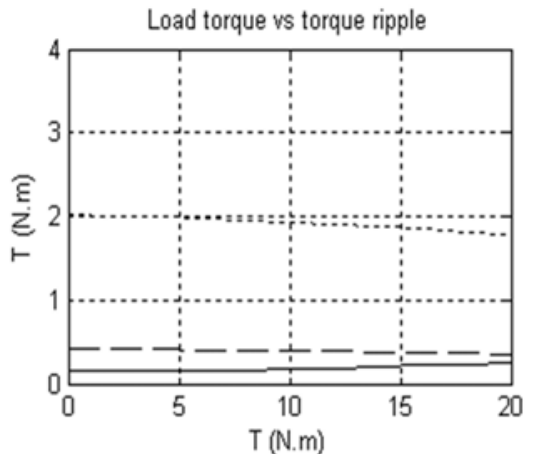

(a)

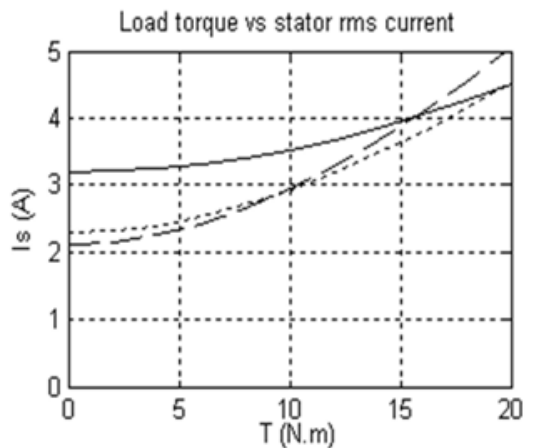

(c)

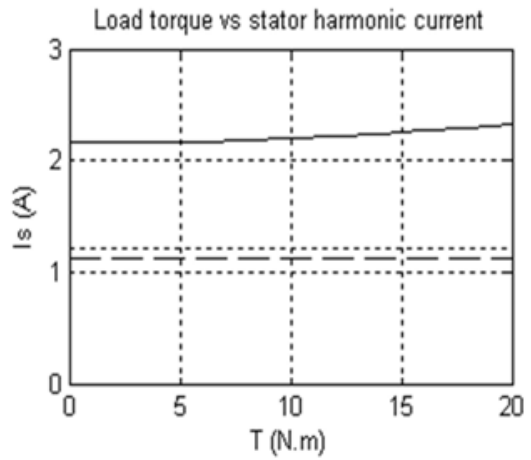

(e)

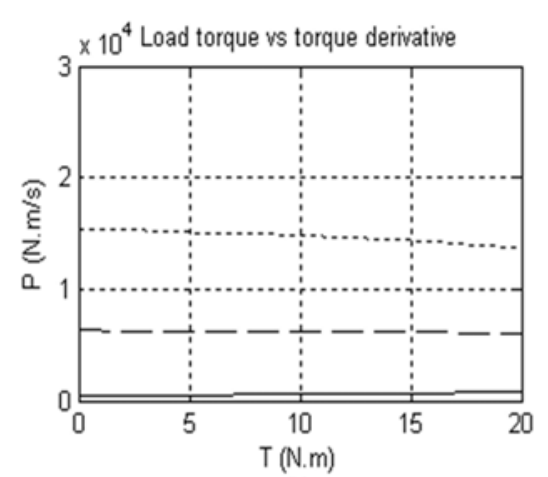

(b)

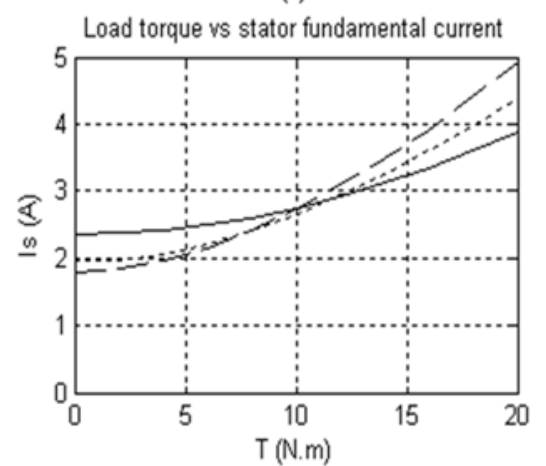

(d)

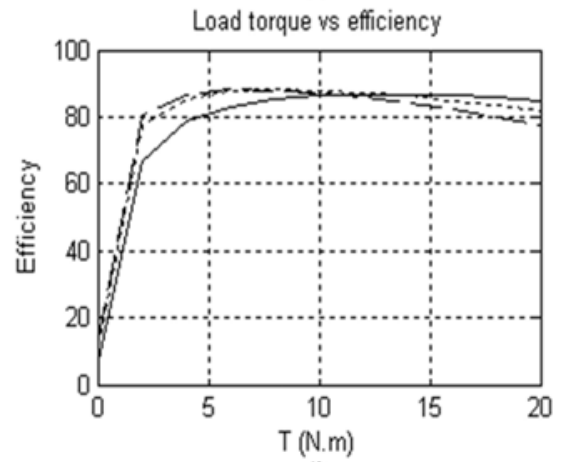

(f)

Figure 5. Variable DC-link simulation results at half the rated voltage and speed for load torque versus torque ripple (a), torque derivative (b), stator RMS current (c), stator fundamental current (d), stator harmonic current (e), and motor efficiency (f).

applications. In both SPM and SPWM, a changing modulation index reduces the voltage's RMS value, while the SHE voltage RMS value is not affected by the modulation index as modulation index affects only the fundamental voltage (Duran et al. 2008; Abdelsalam et al. 2010). This means that SHE only can operate effectively at modulation index one. The alternative solution to avoid such voltage stresses on motor insulation is to use either SPWM or SPM, as this increases a motor's life span. Figure 6e shows that if the modulation index is changed, the SHE has the lowest efficiency with respect to SPWM and SPM due to the large harmonic content. Figure $6 a$ and $b$ show that SPM has the lowest torque ripple and torque derivative peak-peak value compared to both SHE and SPWM. Accordingly, SPM provides a longer lifespan for a motor's rotor and shaft, 

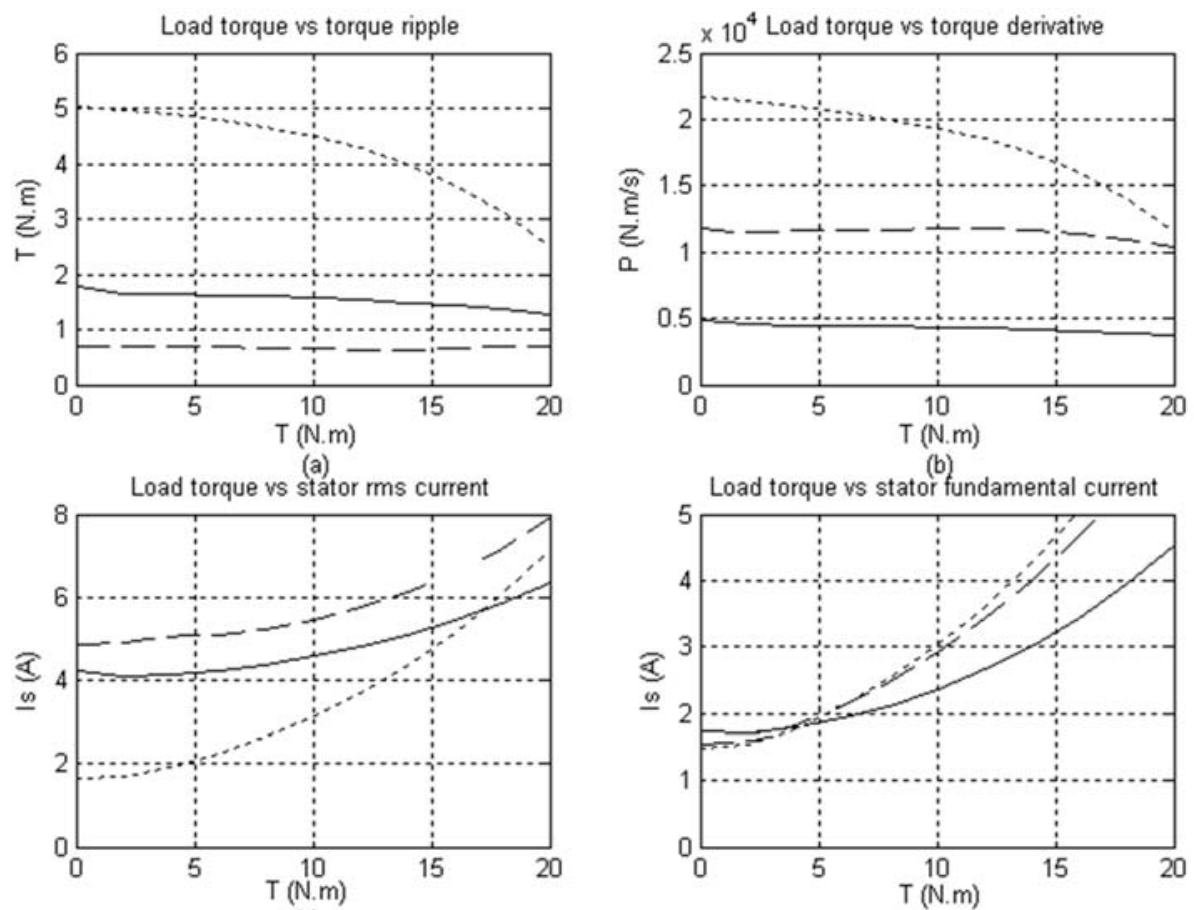

(c)
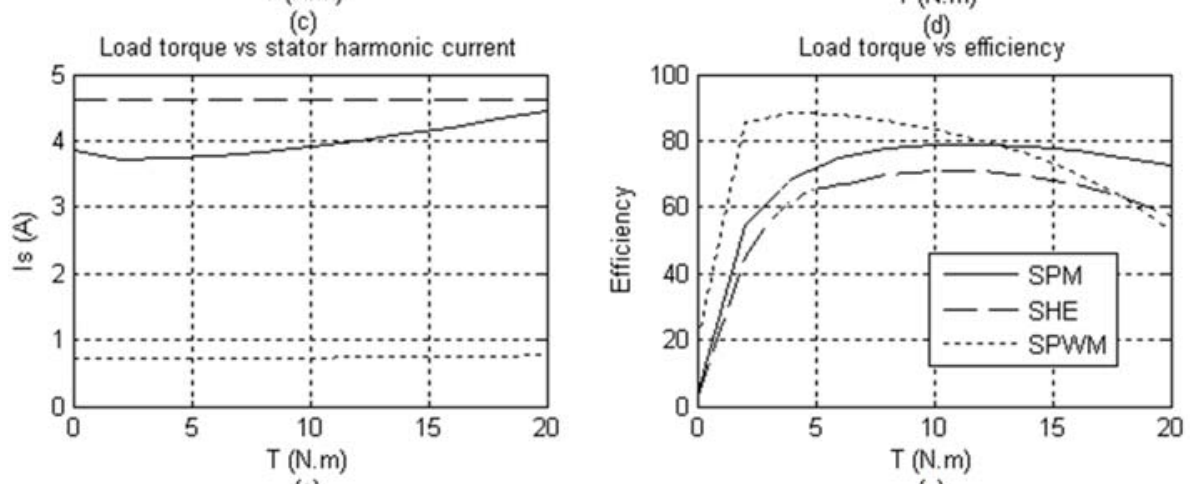

(e)

(f)

Figure 6. Fixed DC-link simulation results at half the rated voltage and speed for load torque versus torque ripple (a), torque derivative (b), stator RMS current (c), stator fundamental current (d), stator harmonic current (e), and motor efficiency (f).

simple algorithm, and simple hardware requirements. Consequently, it is recommended for MV applications. If the motor efficiency is the main concern, the motor controller can switch between SPWM and SPM depending on loading conditions, taking into consideration that both SHE and SPWM have higher switching losses than SPM, especially in MV drives.

From previous results, it is clear that SPWM yields high torque ripples which affect the life span of both a rotor and shaft. Thus, the use of either SPM or SHE is recommended. For cheap and simple alternating current $(\mathrm{AC})$ converters with a fixed DC link, SHE is not the proper choice if a modulation index lower than the value of unity is used. However, if the operation occurs at the unity modulation index, SHE is recommended for light and medium loads while SPM is recommended for heavy loads. From a mechanical lifespan point of view, SPM is the proper choice.

A dynamic performance of the machine was tested during loading conditions. The machine was started unloaded and then, after 0.7 seconds, was loaded by $17 \mathrm{Nm}$ to test for the different control techniques. The fundamental frequency 

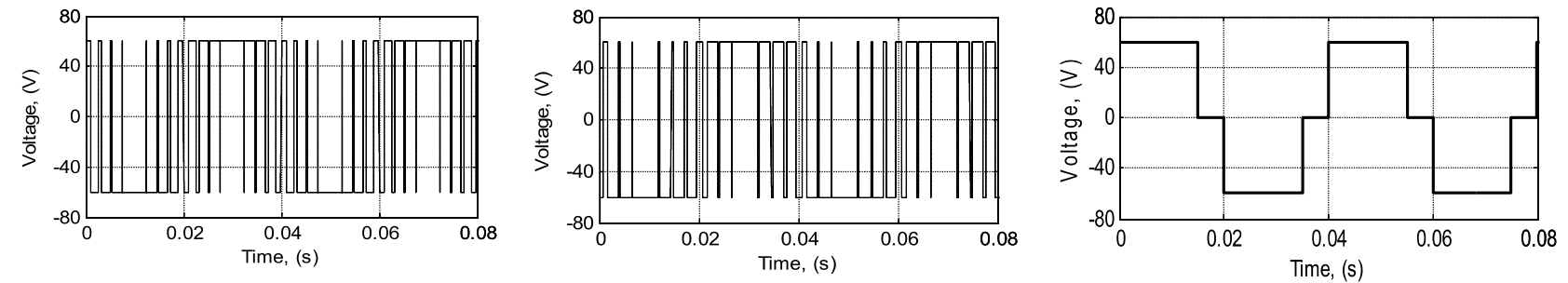

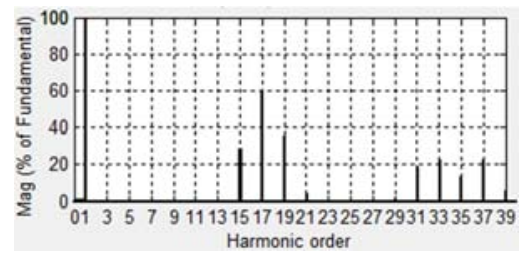

(a)

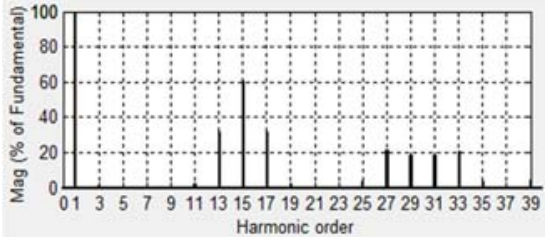

(b)

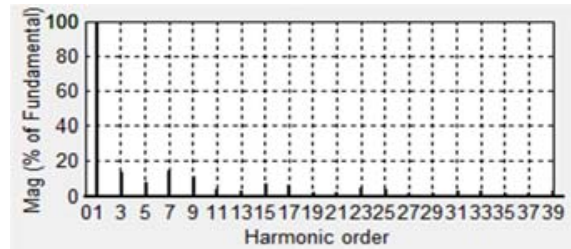

(c)

Figure 7. Phase one motor voltage and its spectrum through the SHE (a), SPWM (b), and SPM (c) techniques.

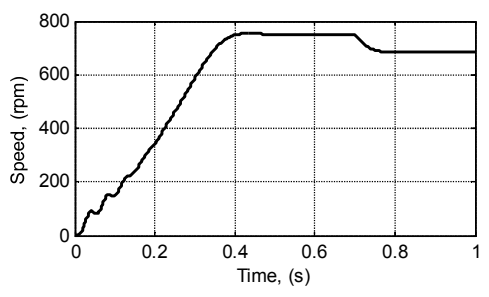

(a)

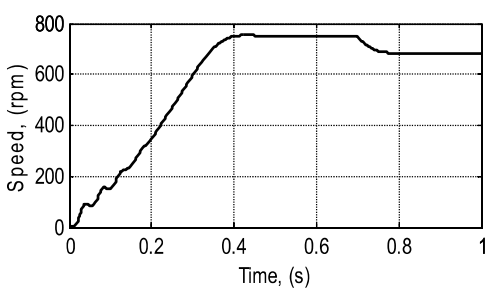

(b)

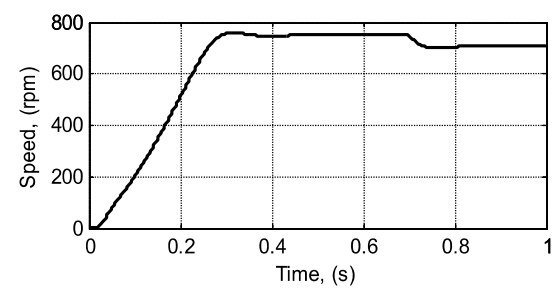

(c)

Figure 8. Motor speed of the SHE (a), SPWM (b), and SPM (c) techniques.

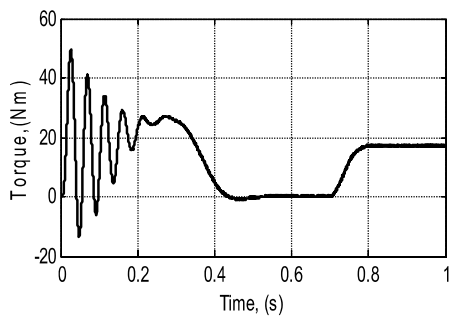

(a)

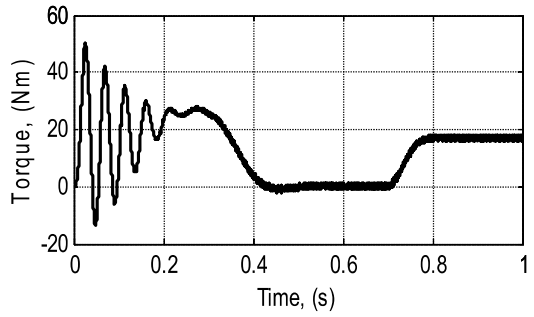

(b)

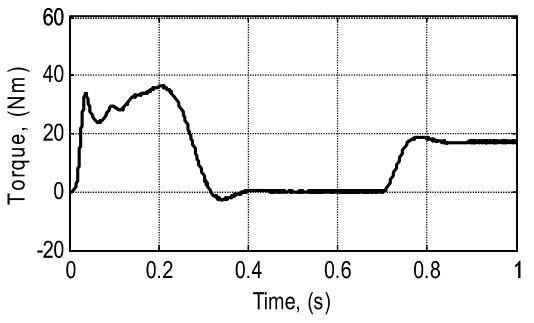

(c)

Figure 9. Motor torque of the SHE (a), SPWM (b), and SPM (c) techniques. 

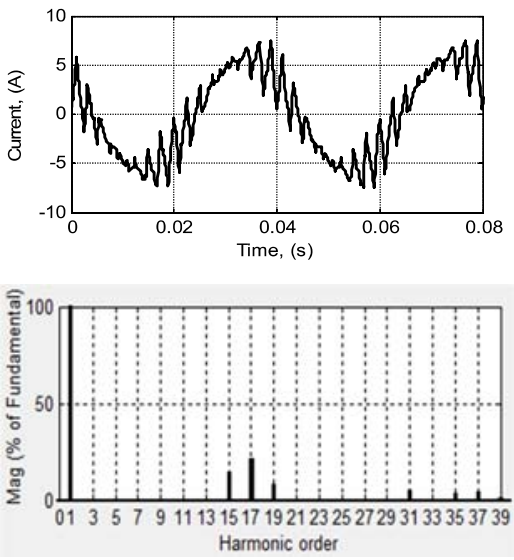

(a)
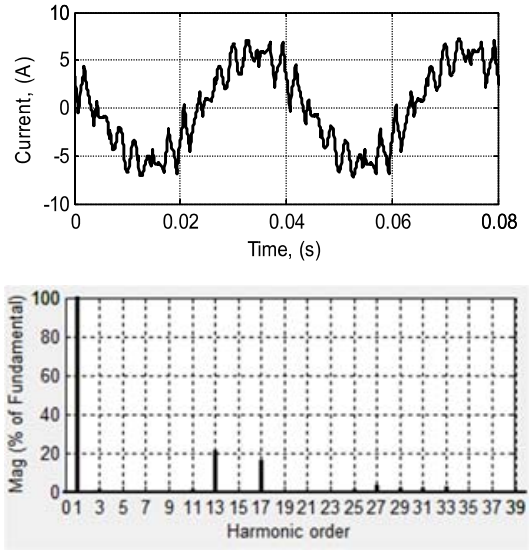

(b)
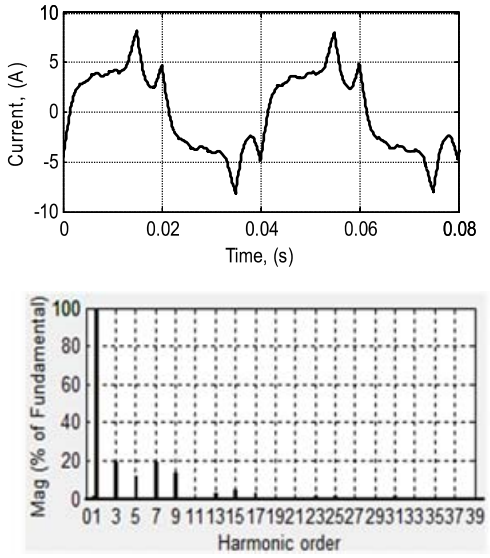

(c)

Figure 10. Phase one of the motor current and its spectrum in the SHE (a), SPWM (b), and SPM (c) techniques.

was adjusted to $25 \mathrm{~Hz}$ and the switching frequency for the SPWM technique was adjusted to $375 \mathrm{~Hz}$. The modulation index was adjusted to 1. Figure 7 shows the applied voltage for two cycles and its spectrum where part a was for the SHE technique, part $b$ was for the SPWM technique, and part c was for the SPM technique. Figure 8 shows the motor speed while Fig. 9 shows the motor torque. Figure 10 shows two cycles for first phase of the motor current during the loading period and its spectrum.

Figure 7a shows that the lowest order harmonic $(\mathrm{LOH})$ displayed on the spectrum was the fifteenth harmonic while in the SPWM, the $\mathrm{LOH}$ was the thirteenth harmonic (Fig. 7b). For SPM technique, all the harmonics were included with lower values and the machine operated with a harmonic injection technique like a square wave supply (Abdelkhalik et al. 2010). Figure 8 shows that the response of the machine to reaching a steady state using the SPM technique was almost half of both the SHE and SPWM techniques. Moreover, the speed decreased witnessed during the loading period was lower than in the other two techniques due to the enhancement of a harmonic injection. This dynamic performance was reflected by torque-time characteristics as is clearly seen in Fig. 9c where the starting oscillation was almost cancelled. Also, Fig. 9b clearly shows that the SPWM gives a high ripple torque as compared to other techniques, confirming the results given in Figs. 4-6. The
SPM technique had the lowest torque ripple. Figure 10 shows the phase one current and its spectrum which follows the spectrum of phase voltage but with different amplitudes.

\subsection{Practical Results}

Figure 11 shows the experimental setup block diagram, while Fig. 12 shows steady-state experimental results under SHE, with a switching frequency of $375 \mathrm{~Hz}$ and the SPM (square wave) at a rated voltage and frequency. SPWM was excluded from this test since it results in high torque ripples. Parts $\mathrm{a}$ and $\mathrm{b}$ shows the currenttorque and torque-efficiency characteristics for the two control techniques, respectively. Figure 12 confirms that, for heavy loads with a phase current $\geq 4.5$ amperes (A), the SP is the preferred choice where the torque/current ratio is increased and efficiency is improved. For medium loads of 3-4.5 A, the SHE technique is better than the SP technique but attains higher torque ripples. Hence, for light and medium loads, the user can use SHE if the efficiency is the target, but if the target is to avoid torque ripple and switching losses, SPM is the better choice.

\section{Conclusions}

This paper introduced a comparative study of different PWM techniques applied to a multiphase machine via multiphase voltage source 


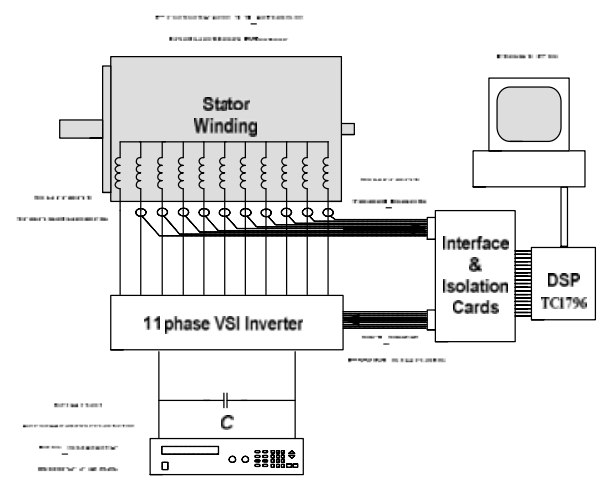

Figure 11. Experimental setup block diagram.
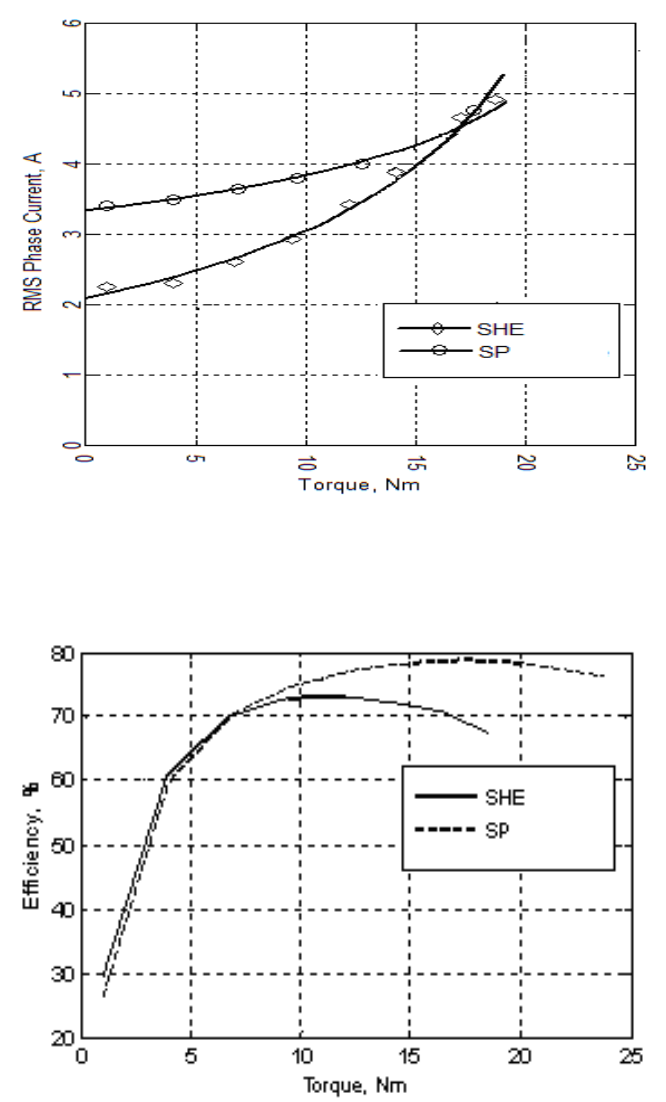

Figure 12. Performance evaluation of SHE and SPM as a square wave (a) Torquecurrent characteristics, and (b) Torque-efficiency characteristics.

inverters; SHE, SPM, and SPWM. The findings show that the SHE technique or SPM wave have advantages over SPWM, such as a lower phase current, reduced torque pulsation, and an increased torque per current ratio. Moreover, a harmonic current enhances motor torque, thereby reducing switching losses that occur due to a lowvalue switching frequency as compared to a SPWM, which demands a high switching frequency in order to obtain an acceptable performance. The SPWM yields high torque ripples which can affect the motor and shaft lifespan. Hence, it is recommended to use either SP or SHE, especially for MV drives. For efficient operation, SHE is recommended only with a unity modulation index because, if the modulation index is lowered, efficiency becomes worse and, in this case, SPM modulation is the choice. In addition, if mechanical lifespan is of concern, SPM is recommended.

\section{References}

Abdelkhalik A, Masoud M, Williams BW (2010), Eleven-phase induction machine: Steady-state analysis and performance evaluation with harmonic injection. IET Electric Power Applications 4(8):670-685.

Abdelkhalik A, Masoud M, Williams BW (2011), Performance evaluation of eleven-phase induction motor using selective harmonic elimination. Proceeding of the 2011 International conference on power engineering, energy and electrical drives, POWERENG'11.

Abdelsalam AK, Masoud MI, Finney SJ, Williams BW (2010), Medium-voltage pulse width modulated current source rectifiers using different semiconductors: Loss and size comparison. IET Power Electronics 3(2):243-258.

Blasko V (2007), A novel method for selective harmonic elimination in power electronic equipment. IEEE Transactions on Power Electronics 22(1):223-228.

Duran MJ, Salas F, Arahal MR (2008), Bifurcation analysis of five-phase induction motor drives with third harmonic injection. IEEE Transactions on Industrial Electronics 55(5):2006-2014.

Espinoza JR, Joos G, Guzman JI, Moran LA, Burgos RP (2001), Selective harmonic elimination and current/voltage control in current/voltage-source topologies: a unified approach. IEEE Transactions on Industrial Electronics 48(1):71-81. 
Levi E (2008), Multiphase electric machines for variable-speed applications. IEEE Transactions on Industrial Electronics 55(5):1893-1909.

Levi E, Bojoi R, Profumo F, Toliyat HA, Williamson S (2007), Multiphase induction motor drives - A technology status. IET Electric Power Applications 1(4):489-516.

Libo Z, Fletcher JE, Williams BW, Xiangning $\mathrm{H}$ (2008), Dual-plane vector control of a five-phase induction machine for an improved flux pattern. IEEE Transactions on Industrial Electronics 55(5):1996-2005.

Parsa L (2005), On advantages of multi-phase

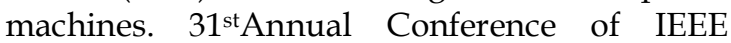
Industrial Electronics Society, IECON,15741579.

Pavithran KN, Parimelalagan R, Krishnamurthy MR (1988), Studies on inverter-fed five-phase induction motor drive. IEEE Transactions on Power Electronics 3(2):224-235.
Pereira LA, Scharlau CC, Pereira LFA, Haffner JF (2006), General model of a five-phase induction machine allowing for harmonics in the air gap field. IEEE Transaction on Energy Conversion 21(4):891-899.

Singh GK (2002), Multi-phase induction machine drive research-A survey. Elsevier, Electric Power Systems Research 61(2):139-147.

Toliyat HA, Lipo TA, White JC (1991), Analysis of a concentrated winding induction machine for adjustable speed drive applications. Part 1. Motor analysis. IEEE Transaction on Energy Conversio 6(4):679-683.

Zhang W, Sun Y (2006), Optimization of output voltage waveform of selective harmonic elimination inverter. International Conference on Power System Technology 1-4. 


\section{Appendix}

Machine ratings

\begin{tabular}{|l|c|c|}
\hline Rated Power & $\mathrm{hp}$ & 3 \\
\hline Number of poles & & 4 \\
\hline Phase voltage & $\mathrm{V}$ & 82 \\
\hline Rated phase current & $\mathrm{A}$ & 4 \\
\hline Rated torque & $\mathrm{Nm}$ & 15 \\
\hline Supply frequency & $\mathrm{Hz}$ & 50 \\
\hline Phase connections & & Star \\
\hline
\end{tabular}

Parameters referred to the stator

\begin{tabular}{|l|l|l|l|l|l|}
\hline$R_{s}$ & $0.74 \Omega$ & $l_{s}$ & \multicolumn{3}{|l|}{$5.3 \mathrm{mH}$} \\
\hline$R_{r 1}$ & 1 & $l_{r 1}$ & $16 \mathrm{mH}$ & $L_{m 1}$ & $140.3 \mathrm{mH}$ \\
\hline$R_{r 3}$ & 1.3351 & $l_{r 3}$ & $21.36 \mathrm{mH}$ & $L_{m 3}$ & $17.97 \mathrm{mH}$ \\
\hline$R_{r 5}$ & 2.7748 & $l_{r 5}$ & $44.4 \mathrm{mH}$ & $L_{m 5}$ & $6.57 \mathrm{mH}$ \\
\hline$R_{r 7}$ & 3.8850 & $l_{r 7}$ & $62.16 \mathrm{mH}$ & $L_{m 7}$ & $3.7 \mathrm{mH}$ \\
\hline$R_{r 9}$ & & $l_{r 9}$ & $79.91 \mathrm{mH}$ & $L_{m 9}$ & $2.4 \mathrm{mH}$ \\
\hline
\end{tabular}

Practical test-rig

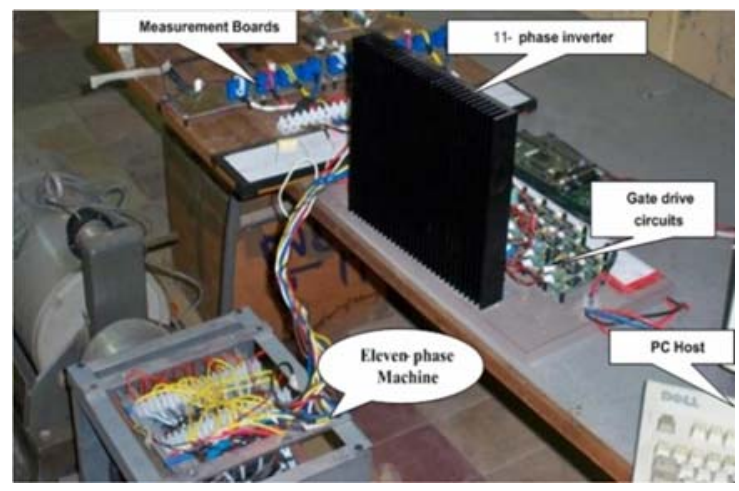


Eleven-phase machine construction

\begin{tabular}{|l|c|c|}
\hline No. of stator slots & & 44 \\
\hline Rotor radius & $\mathrm{mm}$ & 63 \\
\hline Rotor stack length & $\mathrm{mm}$ & 121 \\
\hline Number of rotor slots & & 28 \\
\hline Number of turns per phase & & 80 \\
\hline
\end{tabular}

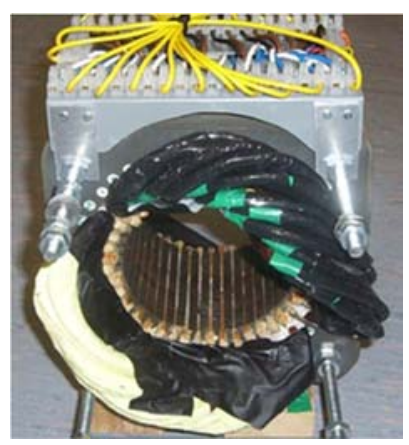

